\title{
Availability and Reliability of Wireless Links in 5G Systems: A Space-Time Approach
}

\author{
Mustafa Emara, Miltiades C. Filippou, Ingolf Karls \\ Next Generation and Standards, Intel Deutschland GmbH, Neubiberg, Germany \\ Email:\{mustafa.emara, miltiadis.filippou, ingolf.karls\}@intel.com
}

\begin{abstract}
Wireless links are characterized by fluctuating quality leading to variable packet error rates which are orders of magnitude higher than the ones of wired links. Therefore, it is of paramount importance to investigate the limitations of using $5 \mathrm{G}$ wireless links for internet of things (IoT) applications. 5G wireless links in IoT need to assure determinism of process flows via realtime communication anytime and anywhere, which is an utmost requirement for multiple verticals like automotive, industrial automation and aerospace. Based on a space-time approach, in this work, we provide novel definitions of wireless link availability and reliability, assuming a number of access points (APs) and end points (EPs) deployed over a fixed area. Our objective is to analyze the availability of a service in both domains. In the space domain, we characterize spatially available areas consisting of all locations that meet a performance requirement with confidence. In the time domain, we propose a channel allocation scheme accounting for the spatial availability of a cell. To emphasize the incurred space-time performance trade-offs, numerical results are presented, also highlighting the effect of different system parameters on the achievable link availability and reliability.
\end{abstract}

Index Terms-5G, availability, reliability, space-time analysis

\section{INTRODUCTION}

Due to the increasing service requirements posed by verticals ready to exploit fifth generation $(5 \mathrm{G})$ wireless communication systems, enhancing existent key performance indicators (KPIs) and defining new ones is inevitable [1]. Two important performance requirements are the reliability and availability of communication, which need to be satisfied in an end-toend manner [2]. Among the established 5G service categories, ultra reliable low latency communication (URLLC) shall pave the way for internet of things (IoT), mission critical services, real-time control and automation for multiple market segments [3]. However, URLLC is accompanied by a plethora of challenges and stringent requirements to ensure an ongoing service with virtually no failures during the operation time. These challenges are currently being addressed by academia [4] and different industry associations/ standardization bodies [1], [5], [6]. For instance, URLLC is envisaged to support a packet failure probability of $10^{-7}$, while introducing latencies of merely up to the few milliseconds range [3]. Since such performance demands are challenging for legacy wireless networks, a paradigm shift from the conventional network architecture is inevitable.

In both recent 3rd Generation Partnership Project (3GPP) specifications and academic research works, URLLC service performance has been mainly evaluated via investigating metrics such as packet error ratio, latency and jitter [1],
[3]. These metrics, though fundamentally meaningful from the radio communication perspective, need to be looked at together with service demands from a vertical-specific point of view (e.g., availability of a service and reliability of the operation). Consequently, such service-specific metrics need to be first well defined, understood and then mapped to the wireless system's parameters, prior to evaluating system-wide feasibility of the focused service/operation. Conceptually, this novel system view aims to unlock the potential of running wireless services quasi-deterministically, thus, enabling a finegrained system analysis [4].

\section{A. Related Work}

To the best of our knowledge, adopting definitions of service-tailored link availability and reliability for wirelessbased systems has not yet been expressed adequately. In [7], the authors have proposed a new definition of spatial availability, as the ratio of the mean covered area to the geographical area of a given access point (AP). Nevertheless, an interference-free scenario was considered and no insights on the time evolution of communication availability were provided. Additionally, in [8], the authors proposed a reliability metric, consisting of two components: the temporal availability and the probability to overcome a received power threshold, however, for a single cell scenario. Furthermore, the authors in [9] summarized main definitions from reliability theory [10], and presented an automation-based use case exploiting multi-link connectivity. Nevertheless, spatial availability analysis was not considered at all. In addition, the authors in [4], provided an tutorial-like overview, introducing different challenges and solution proposals for URLLC services. Although quite insightful, this work did not touch upon the concepts of time and space availability. Finally, [11] proposed a new protocol enabling precise synchronization for wireless time sensitive networking. Although no definitions of reliability or availability were discussed in this work, the proposed framework aims to achieve operation determinism in wireless systems.

\section{B. Contributions}

Motivated by the above, in this paper, concentrating on both space and time domains, we make a first attempt to bridge the gap between traditional radio link KPIs and servicelevel KPIs by providing an insight on the availability and reliability of wireless links in $5 \mathrm{G}$ systems. The proposed 
framework aims to indicate which locations in a given area would overcome a performance threshold over a specific time window with a guaranteed level of confidence. In further detail, the contributions of the paper are the following

- Focusing on a radio access technology (RAT)-agnostic wireless system, we propose the definition of a new, stochastic quantity to measure the spatial availability of a wireless link for a service-relevant confidence level.

- Capitalizing on the proposed definition of spatial availability, we propose a novel resource allocation approach dependent on the spatially available area of a given AP and based on the concept of resource provisioning. In addition, transient and steady-state analysis of the system's temporal availability is performed and the relation between temporal availability and reliability is highlighted.

- We present numerical evaluations, highlighting the different effects of system parameter values on spatial availability, as well as on temporal availability and reliability. In addition, we show the relation between spatial and steady state temporal availability.

\section{Notation}

Throughout this paper, we adopt the following notation. Matrices and vectors are represented as upper-case and lowercase boldface letters $(\boldsymbol{A}, \boldsymbol{a})$, respectively. Also, $f_{\boldsymbol{i}}$ denotes the value of quantity $f$ at location $\boldsymbol{i}$ of Cartesian coordinates $(x, y)$. The operation $\lceil a\rceil$ represents the rounding of $a, \mathbb{1}(a)$ is the indicator function, which equals 1 if $a$ is true and 0 otherwise and $\min (a, b)$ is the minimum operator of $a$ and $b$. Moreover, $\mathbb{P}[A]$ is the probability of event $A, \mathbb{E}[X]$ is the expectation of random variable $X, M_{X}($.$) is the moment$ generating function (MGF) of $X$, and finally, the operation $d(\boldsymbol{x}, \boldsymbol{y})$ represents the Euclidean norm between $\boldsymbol{x}$ and $\boldsymbol{y}$.

\section{SyStem MODEL}

In this paper, a downlink wireless system, consisting of $N$ single-antenna access points (APs) of equal transmit power is deployed over a two-dimensional bounding box (e.g., a factory floor). It is assumed that each spatial deployment realization of the $N$ APs is denoted as $\Phi$ (i.e., $\left\{l \mid\left(x_{l}, y_{l}\right) \in \mathbb{R}^{2}, l \in \Phi\right\}$ ). Without loss of generality, the proposed system model can be applied to different communication systems. A multitude of end points (EPs), like personal tablets, control units, sensors or actuators, are being served via wireless links, as shown in Fig. 11 Each AP has access to $M$ orthogonal channels that can be used for downlink transmission, so as for the EPs to fulfill their service requests. At the EP side, service requests form an arrival process which follows a Poisson distribution with an average arrival rate denoted by $\lambda$, whereas the service time of a downlink transmission follows an exponential distribution with an average service rate of $\mu$. A frequency reuse factor of one is assumed in this work, which translates to the potential presence of inter-cell interference among the $N$ APs. From a joint deployment and connectivity point of view, the cell's connectivity region (i.e., the shaded area in Fig. 1, also named as Voronoi cell) represents the geographical area in which a

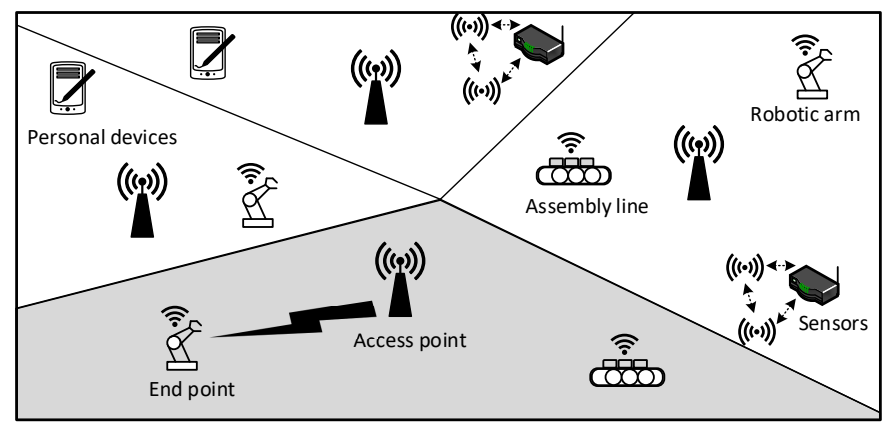

Fig. 1: The investigated system model consisting of different EPs types, each served by a single AP. The shaded area represents the connectivity region of an AP.

wireless link can be established between an EP and its closest AP. Equivalently, Voronoi cells are shaped by applying an EPAP connectivity rule based on a minimum pathloss criterion.

An important metric to evaluate quality of service (QoS) in interference-limited scenarios, is the signal to interference ratio (SIR) [12]. The SIR of a generic EP located at point $i$ and served by an AP located at point $j$, where $i$ and $j \in \mathbb{R}^{2}$, is computed as

$$
\mathrm{SIR}_{\boldsymbol{i}, \boldsymbol{j}}=\frac{P_{\mathrm{Tx}} h_{\boldsymbol{i}, \boldsymbol{j}} \mathcal{L}(\boldsymbol{i}, \boldsymbol{j}, \eta)}{\sum_{\boldsymbol{k} \in \mathcal{I}} P_{\mathrm{Tx}} h_{\boldsymbol{i}, \boldsymbol{k}} \mathcal{L}(\boldsymbol{i}, \boldsymbol{k}, \eta)},
$$

where $P_{\mathrm{Tx}}$ is the transmit power of the $\mathrm{AP}, h_{\boldsymbol{i}, \boldsymbol{j}}, h_{\boldsymbol{i}, \boldsymbol{k}}, k \in \mathcal{I}$ are the small-scale fading parameters, $\eta$ is the pathloss exponent and $\mathcal{I}$ represents the set of all the $N-1$ interfering APs. The function $\mathcal{L}($.$) computes the pathloss attenuation between$ two points $\boldsymbol{i}, \boldsymbol{j} \in \mathbb{R}^{2}$ as follows: $\mathcal{L}(\boldsymbol{i}, \boldsymbol{j}, \eta)=(d(\boldsymbol{i}, \boldsymbol{j}))^{-\eta}$. It should be noted that each AP is aware of the locations of interfering APs. Additionally, the fast fading parameters are assumed to be Rayleigh distributed with unit average power, i.e., for every AP-EP link, $h \approx \exp (1)$ and the fast fading effects are assumed non-correlated among the various links. To investigate the guaranteed performance of a given AP-EP link, we introduce a new binary evaluation metric, $\Omega_{\boldsymbol{i}, \boldsymbol{j}}(\theta, \alpha, \Phi)$, having as a decision criterion the probability for a wireless link to achieve a given SIR threshold $\theta$ with a predetermined confidence level $\alpha$ for a given AP deployment realization $\Phi$ [13]. This quantity is mathematically expressed as follows

$$
\Omega_{\boldsymbol{i}, \boldsymbol{j}}(\theta, \alpha, \Phi)=\mathbb{1}\left(\mathbb{P}\left[\operatorname{SIR}_{\boldsymbol{i}, \boldsymbol{j}} \geq \theta\right] \geq \alpha\right) .
$$

This metric will be exploited in the coming section for defining the spatial, service-relevant availability of a wireless link.

\section{Availability Analysis: Spatial Domain}

Throughout this section, we aim to project the wellestablished definitions of time-domain availability and reliability to the spatial domain. Temporally, instantaneous availability of a system is the probability of the system being operational at a given time instant [10], whereas, in the space domain, as introduced in [7], the spatial availability $A_{s}$, defines the locations on a given Euclidean plane, where the system is operational. The region of operation was modeled 
as the cell's circular coverage area in [7], due to the lack of interference, whereas, in [14], the authors proposed multiple criteria for the definition of spatial availability. Inspired by these two works, in this section we propose a new, servicerelated definition of spatial availability, taking into account the confidence level of surpassing a predefined SIR threshold. We present the following definition:

$(\boldsymbol{\theta}, \boldsymbol{\alpha})$-availability. Any EP located at $\boldsymbol{i}$ and served by an AP located at $\boldsymbol{j}$ is labeled as $(\theta, \alpha)$-available, if $\Omega_{\boldsymbol{i}, \boldsymbol{j}}(\theta, \alpha, \Phi)=$ $1, \boldsymbol{j} \in \Phi, \boldsymbol{i}, \boldsymbol{j} \in \mathbb{R}^{2}$, and non-available otherwise.

Given an AP deployment $\Phi$ and accumulating all EPs possible locations $\boldsymbol{z}, \boldsymbol{z} \in \mathbb{R}^{2}$ which satisfy the $(\theta, \alpha)$ spatial availability criterion $\Omega_{\boldsymbol{z}, \boldsymbol{j}}(\theta, \alpha, \Phi)$ when connected to an AP located at point $\boldsymbol{j}$, we obtain the following $(\theta, \alpha)$-available region $\mathcal{D}_{j}$ as follows

$$
\mathcal{D}_{j}=\left\{\boldsymbol{z} \in \mathbb{R}^{2} \mid \boldsymbol{z} \in \mathcal{D}_{\boldsymbol{j}}, \Omega_{\boldsymbol{z}, \boldsymbol{j}}(\theta, \alpha, \Phi)=1, \boldsymbol{j} \in \Phi\right\} .
$$

Thus, the proposed spatial availability for an AP located at $\boldsymbol{j}$, can be formulated as

$$
A_{s}(\boldsymbol{j})=\min \left(1, \frac{\operatorname{Area}\left(\mathcal{D}_{\boldsymbol{j}}\right)}{\operatorname{Area}\left(\mathcal{V}_{\boldsymbol{j}}\right)}\right)=\min \left(1, \frac{\left|\mathcal{D}_{\boldsymbol{j}}\right|}{\left|\mathcal{V}_{\boldsymbol{j}}\right|}\right)
$$

where the minimum operator accounts for cases where the $(\theta, \alpha)$-available area is larger than geographical area of the AP (in such cases $A_{s}(\boldsymbol{j})=1$ ) and $\mathcal{V}_{\boldsymbol{j}}$ is the collection of points constituting the Voronoi cell of the $j$-th AP. In addition, eq. (4) can be expanded as

$$
\min \left(1, \frac{\int_{\boldsymbol{z} \in \mathbb{R}^{2}} \mathbb{1}\left(\boldsymbol{z} \in \mathcal{D}_{\boldsymbol{j}}\right) \mathrm{d} \boldsymbol{z}}{\frac{1}{2}\left|\sum_{l}^{g-1} x_{l} y_{l+1}+x_{q} y_{q}-\sum_{l}^{g-1} x_{l+1} y_{l}-x_{q} y_{q}\right|}\right),
$$

where $g$ is the number of edges and $\left(x_{l}, y_{l}\right)$ are the Cartesian coordinates of the $l$-th vertex of the Voronoi cell. The denominator in eq. (4) is obtained by applying the wellknown shoelace algorithm that computes the area of a Voronoi polygon with $g$ edges [7].

In order to compute the area of set $\mathcal{D}_{j}$, its boundary needs to be specified. In other words, focusing on an AP, all the points satisfying the SIR threshold $\theta$ with confidence level $\alpha$ are sought. One can thus expand eq. (2) [15], for a given spatial deployment ( $\Phi$ is dropped for simplicity) as follows

$$
\begin{aligned}
\Omega_{\boldsymbol{z}, \boldsymbol{j}}(\theta, \alpha) & =\mathbb{1}\left(\mathbb{P}\left[\frac{P_{\mathrm{Tx}} h_{\boldsymbol{z}, \boldsymbol{j}} \mathcal{L}(\boldsymbol{z}, \boldsymbol{j}, \eta)}{\sum_{\boldsymbol{k} \in \mathcal{I}} P_{\mathrm{Tx}} h_{\boldsymbol{z}, \boldsymbol{k}} \mathcal{L}(\boldsymbol{z}, \boldsymbol{k}, \eta)} \geq \theta\right] \geq \alpha\right), \\
& \stackrel{(a)}{=} \mathbb{1}\left(\mathbb{E}\left[\exp \left(\frac{-\theta}{\mathcal{L}(\boldsymbol{z}, \boldsymbol{j}, \eta)} \sum_{\boldsymbol{k} \in \mathcal{I}} \mathcal{L}(\boldsymbol{z}, \boldsymbol{k}, \eta)\right] \geq \alpha\right),\right. \\
& \stackrel{(b)}{=} \mathbb{1}\left(\prod_{k \in \mathcal{I}} M_{h}\left(\frac{-\theta}{\mathcal{L}(\boldsymbol{z}, \boldsymbol{j}, \eta)} \mathcal{L}(\boldsymbol{z}, \boldsymbol{k}, \eta)\right) \geq \alpha\right), \\
& \stackrel{(a)}{=} \mathbb{1}\left(\prod_{k \in \mathcal{I}} \frac{1}{1+\frac{\theta}{\mathcal{L}(\boldsymbol{z}, \boldsymbol{j}, \eta)} \mathcal{L}(\boldsymbol{z}, \boldsymbol{k}, \eta)} \geq \alpha\right),
\end{aligned}
$$

where $(a)$ follows since fast-fading channels are assumed to be exponentially distributed and $(b)$ is the MGF of an exponentially distributed random variable. Since the APs transmit with equal power, the final expression of $\Omega_{\boldsymbol{z}, \boldsymbol{j}}(\theta, \alpha)$ is transmit power independent [12]. (a)

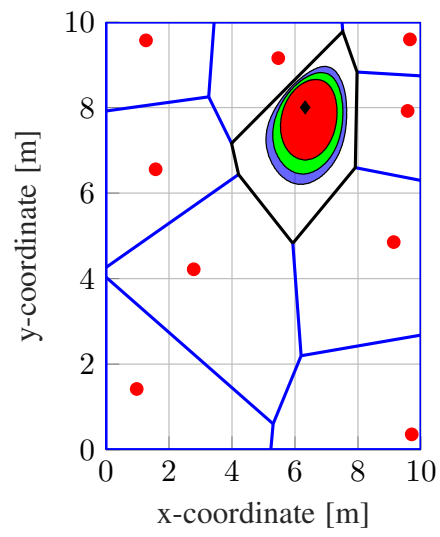

(b)

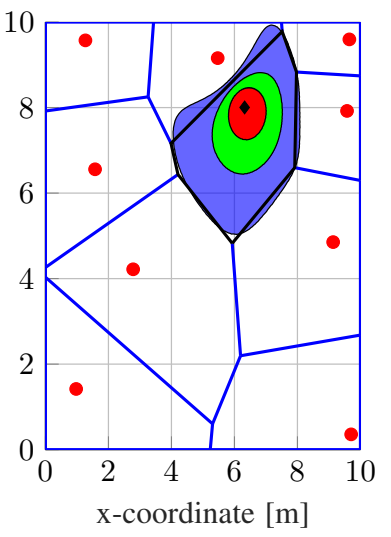

Fig. 2: $(\theta, \alpha, \Phi)$-available regions for a deployment with $N=$ 10 APs focusing on a generic AP (its Voronoi border drawn in black) (a) $\theta=0 \mathrm{~dB}$ and $\alpha=(0.7,0.8,0.9)$ and (b) $\theta=$ $(-10,0,10) \mathrm{dB}$ and $\alpha=0.8$.

Expression eq. (6) can be utilized to define the boundary of $\operatorname{Area}\left(\mathcal{D}_{j}\right)$ by substituting inequality by pure equality. However, since this boundary is hardly tractable in closed form, to obtain quantitative results, we resort to a bisection-based algorithm to approximate the size of this area to be then used in eq. (4). A visualization of the computed regions is shown in Fig. 2, where different combinations of $(\theta, \alpha)$ are considered for a given AP. It is observable that in Fig. 2 (b), the $(-10 \mathrm{~dB}, 0.8)$ region is not convex, due to the interference imposed by the closest interfering AP, which reveals that, along with $(\theta, \alpha)$, the number and location of deployed and, consequently, interfering APs is expected to highly affect the spatial availability. Thorough investigation on the effect of $(\theta, \alpha)$ on spatial availability for random AP deployments will be considered in Section $\mathrm{V}$.

\section{AVAilability Analysis: Temporal Domain}

Having analyzed the spatial availability metric in the previous section, and since our objective is to propose a unified, space-time availability framework useful to URLLC systems, in this section we concentrate on the time domain.

\section{A. Resource Partitioning}

As explained in Section III each AP has $M$ channels that can be accessible by the EPs in its Voronoi region. To account for the spatial availability $A_{s}$ as defined in Section III we propose a spatial availability-proportional channel allocation scheme. According to this scheme, since $A_{s}$ decomposes the Voronoi region of the AP into two regions, the number of channels to be utilized by EPs located in the $(\theta, \alpha)$-available and non-available regions of an AP located at point $j$ can be, respectively, written as

$$
M_{a}(\boldsymbol{j})=\left\lceil A_{s}(\boldsymbol{j}) M\right\rceil, M_{n}(\boldsymbol{j})=M-M_{a}(\boldsymbol{j}) .
$$

As a result of the proposed policy, assuming that service requests arrive uniformly in space, when the spatial availability ratio $A_{s}$ is low, a few channels will be allocated to 


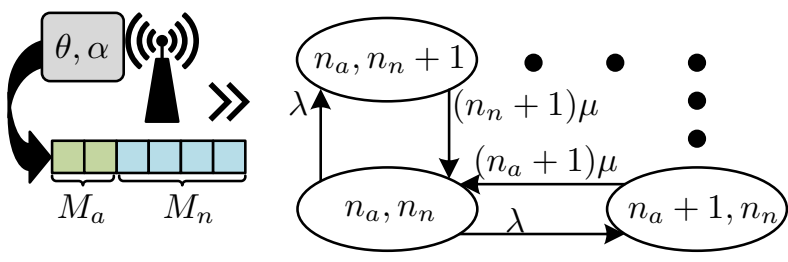

Fig. 3: Resources partitioning based on $A_{s}$ along with part of the two dimensional birth/death Markov process.

the few evolving requests coming from the $(\theta, \alpha)$-available region, while, the majority of channels will be allocated to the (possibly many) requests coming from the $(\theta, \alpha)$ non-available region.

\section{B. Temporal Analysis}

In order to model the time-dependent status of the resources at a generic AP, we resort to a continous time Markov chain (CTMC) model that captures the number of idle/busy channels as time evolves. To also capture the decomposition of service requests into two sets (spatially available/non-available), a two dimensional CTMC is employed, where the first dimension represents the number of EPs being served within the $(\theta, \alpha)$ available region of the AP, and the other dimension represents the number of EPs in the rest of the geographical cell region. Fig. 3 presents the proposed framework, where $n_{a}$ and $n_{n}$ are generic numbers of EPs being served in the two mentioned regions. Such a model leads to a finite birth/death Markov process, where the total number of states is limited by the total number of channels and all possible partitioning options. Thus, for a given channel allocation, the set of feasible states can be represented as

$$
\begin{aligned}
\mathcal{S}= & \left\{\left(n_{a}, n_{n}\right) \mid 0 \leq n_{a} \leq M_{a}, 0 \leq n_{n} \leq M_{n},\right. \\
& \left.M_{a}+M_{n}=M\right\},
\end{aligned}
$$

where the total number of states is $|\mathcal{S}|=\left(M_{a}+1\right)\left(M_{n}+1\right)$, since a number of $n$ channels will lead to $n+1$ states. Based on the above described model, the temporal availability is defined as the probability of at least one channel being available for a new request. As a result, the set of temporally available states for the $(\theta, \alpha)$-available and non-available regions are $\mathcal{A}^{a}=\left\{\left(n_{a}, n_{n}\right), n_{a}=\left\{0,1, \cdots, M_{a}-1\right\}\right\}$ and $\mathcal{A}^{n}=\left\{\left(n_{a}, n_{n}\right), n_{n}=\left\{0,1, \cdots, M_{n}-1\right\}\right\}$, respectively. The state equations can be vectorized as $\boldsymbol{\tau}(t)=$ $\left\{\tau_{1}(t), \tau_{2}(t), \cdots, \tau_{|\mathcal{S}|}(t)\right\}$, where $\tau_{l}(t)$ is the probability of the system being in the $l$-th state at time instant $t$. Resorting to the matrix notation and using the Kolmogorov forward equations [10], the state probabilities can be computed by solving the following equation

$$
\frac{\mathrm{d}}{\mathrm{d} t} \boldsymbol{\tau}(t)=\boldsymbol{\tau}(t) \boldsymbol{Q}
$$

where the infinitesimal generator (i.e., transition rate) matrix is denoted by $Q$, with dimension $|\mathcal{S}| \times|\mathcal{S}|$. To compute the system's temporal availability, one needs to solve eq. (9). We adopted a similar approach as in [16], based on the uniformization method [17], where the solution of eq. (9), for a given initial state probability (i.e., $t=0$ ), denoted by $\tau(0)$, can be rewritten as

$$
\begin{gathered}
\boldsymbol{\tau}(t)=\boldsymbol{\tau}(0) \mathrm{e}^{\boldsymbol{Q} t}=\boldsymbol{\tau}(0) \sum_{i=0}^{\infty} \frac{(\boldsymbol{Q} t)^{i}}{i !}, \\
\stackrel{(a)}{=} \boldsymbol{\tau}(0) \mathrm{e}^{-q t} \sum_{i=0}^{\infty} \frac{(q t)^{i}}{i !} \boldsymbol{R}^{n},
\end{gathered}
$$

where (a) follows from the introduction of $\boldsymbol{R}=\boldsymbol{I}+\frac{1}{q} \boldsymbol{Q}, \boldsymbol{I}$ is the identity matrix and $q$ is a number satisfying $q \geq \max \left(q_{i i}\right)$, where $q_{i i}$ are the diagonal elements of $\boldsymbol{Q}$. To numerically solve eq. (10), the summation must be truncated at level $N_{c}$ as shown in [16]. In order to obtain the system's temporal availability at a given time instant $t$, one needs to consider all the available states as follows

$$
A_{t}^{u}(t)=\sum_{i \in \mathcal{A}^{u}} \boldsymbol{\tau}_{i}(t), u \in\{a, n\}
$$

where index $u \in\{a, n\}$ represents the $(\theta, \alpha)$-available and non-available regions, respectively.

1) Reliability Analysis: Another important metric for the temporal analysis is the system's temporal reliability $R(t)$ [9], which is defined as the probability that the system is operational during time interval $[0, t]$. Such a definition can be employed in the studied CTMC model, by forcing the system to remain in an unavailable state once it reaches one. In other words, the transition rate from unavailable state is set to zero [9]. Such a modification leads to a modified infinitesimal generator matrix $\hat{Q}$ and eq. (9) can be re-expressed as

$$
\frac{\mathrm{d}}{\mathrm{d} t} \hat{\boldsymbol{\tau}}(t)=\hat{\boldsymbol{\tau}}(t) \hat{\boldsymbol{Q}}
$$

where $\hat{\tau}(t)$ corresponds to state probability of the modified CTMC. Accordingly, the system's temporal reliability is computed as

$$
R^{u}(t)=\sum_{i \in \mathcal{A}^{u}} \hat{\boldsymbol{\tau}}_{i}(t), u \in\{a, n\} .
$$

As it will be numerically shown later, the system reliability is always upper bounded by its time availability (i.e., $A_{t}(t) \geq$ $R(t)$ ), since for a repairable system, transition rates from a failed state are non-zero.

2) Steady State Analysis: Another interesting metric relevant to temporal analysis is the steady state time availability, which is time independent and can be interpreted as the average operating time [10]. Mathematically [9], it can be represented as

$$
A_{t}^{u}=\lim _{t \rightarrow \infty} A_{t}^{u}(t)=\sum_{i \in \mathcal{A}^{u}} \boldsymbol{\tau}_{i}=\sum_{i \in \mathcal{A}^{u}} \frac{\rho_{i}}{i !}\left(1+\sum_{l=1}^{M_{u}} \frac{\rho^{l}}{l !},\right),
$$

where $u \in\{a, n\}$ and $\rho=\frac{\lambda}{\mu}$ represents the arrival to service rate ratio.

\section{Simulation Results}

The objective of this section is to highlight how different wireless system parameters affect the system's space-time availability and reliability, as defined in Sections III and IV The values of the involved system parameters are provided in Table I, unless stated otherwise. 
TABLE I: Simulation Parameters.

\begin{tabular}{|l|l|}
\hline Parameter & value \\
\hline \hline Deployment area & $100 \mathrm{~m}^{2}$ \\
Pathloss exponent $(\eta)$ & 4 \\
Total channels $(M)$ & 10 \\
Initial state probability $(\boldsymbol{\tau}(0))$ & $\mathbf{0}$ \\
Requests arrival rate $(\lambda)$ & 8 packets/sec \\
Requests service rate $(\mu)$ & 1 packet/sec \\
Spatial realizations & 10000 \\
\hline
\end{tabular}

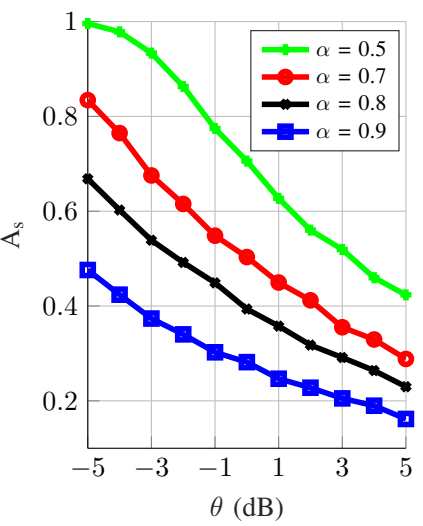

(b)

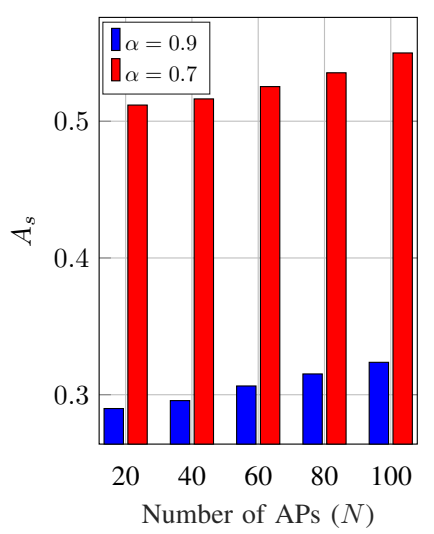

Fig. 4: Spatial availability as a function of (a) $(\theta, \alpha)$ parameters (b) number of APs for $\theta=0 \mathrm{~dB}$.

\section{A. Spatial Analysis}

In Section $\amalg$ a framework for proposing $(\theta, \alpha, \Phi)$-available regions was introduced. As one would expect, the values selected for the mentioned parameters highly affect the achieved spatial availability, thus, to ensure an average insight over all possible AP locations, spatial averaging over a large number of deployments was conducted. In Fig. 4, we highlight the effect of parameters $\theta$ and $\alpha$ along with the number of APs on spatial availability. First, in Fig. (4h), the spatial availability $A_{s}$ of a randomly selected AP is plotted as a function of $\theta$ for different confidence levels, $\alpha$. As expected, for increasing values of $\theta$ (or $\alpha$ ), the spatial availability of that AP decreases, as the equivalent $(\theta, \alpha)$-available region reduces.

Second, in Fig. (4b), $A_{s}$ is depicted as a function of the number of deployed APs for two different confidence levels, when $\theta=0 \mathrm{~dB}$. The monotonically increasing fashion of $A_{s}$ as a function of $N$ for a given value of $\alpha$ is explained as follows: as the system becomes more densified with APs, the Voronoi area of each AP decreases due to the application of a distancebased association criterion and so does also its $(\theta, \alpha)$-available region, due to larger interference received. However, the latter region is less affected compared to the former, due to the stochastic nature of the region forming criterion together with the applied bisection-based approach for computing $(\theta, \alpha)$ available regions.

\section{B. Temporal Analysis}

Based on the presented metrics in Section IV, we investigate, in what follows, the temporal availability and reliability for the proposed access scheme. In Fig. 5, the system's

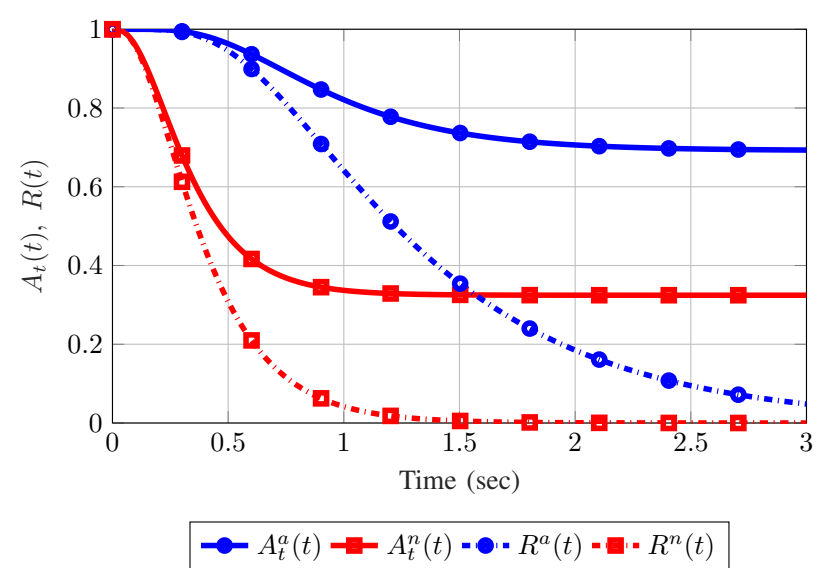

Fig. 5: Temporal availability and reliability of $(\theta, \alpha)$-available and non-available regions for $A_{s}=0.7$.

transient analysis is presented for $A_{s}=0.7$. Due to the spatially-dependent channel allocation proposed in eq. (7), the time availability, $A_{t}^{a}(t)$ (reliability $R^{a}(t)$ ) for a request originating from the $(\theta, \alpha)$-available region should be higher than the time availability $A_{t}^{n}(t)$ (reliability $R^{n}(t)$ ) of the $(\theta, \alpha)$-non available region. This is explained due to the larger number of channels that can be utilized for the available region. It is noticeable that at $t=0$, all channels are available, thus, leading to time availability and reliability equal to one. Additionally, numerical results confirm that the time reliability is upper bounded by time availability, as well as that such a bound is time-dependent since it loosens over time till a maximum performance gap is reached which is then fixed over time.

In Fig. (6), the steady state analysis is illustrated for varying values of the arrival to service ratio $\rho$. As $\rho$ increases, the steady state temporal availability decreases; this occurs due to the fact that the available channels are less in such regimes. Also, as explained earlier, as a result of the adopted channel access scheme, larger values of spatial availability lead to higher time availability. It is, therefore, concluded that $\rho$ is a fundamental performance limitation factor, as for extremely large values of it, even a $100 \%$ spatial availability is unable to be translated to high time availability.

\section{Joint Analysis}

Finally, in Fig. (7), the relationship between the steadystate time availability and the spatial availability is presented for different total channel numbers, $M$. First, we observe a symmetric time availability performance for a fixed $M$ between the $(\theta, \alpha)$-available and non-available regions, due to the proposed channel allocation scheme. For $A s=0.5$, the number of channels allocated to each region will be the same, hence, leading to an identical time availability performance, as requests arrive uniformly in space. Additionally, fixing the value of $A_{s}$, time availability increases together with $M$. This result intuitively emphasizes the role of redundancy and provisioning in wireless systems. Equivalently, through our proposed space-time analysis, the minimum total number of channels needed to achieve a targeted temporal availability level can be identified. To further highlight this, a steady state 


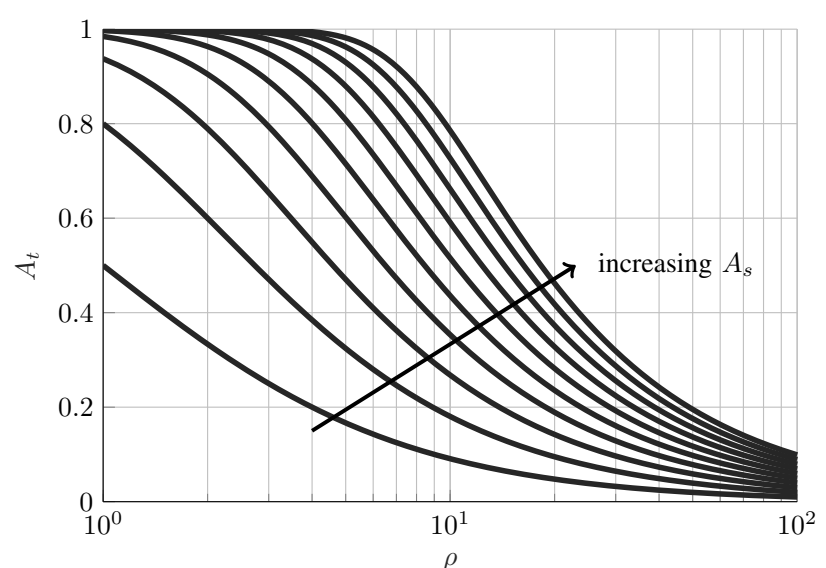

Fig. 6: Steady state time availability, as a function of request arrivals to service rate ratio, for increasing values of spatial availability $\left(A_{s}\right)$ ranging from 0.1 to 1 .

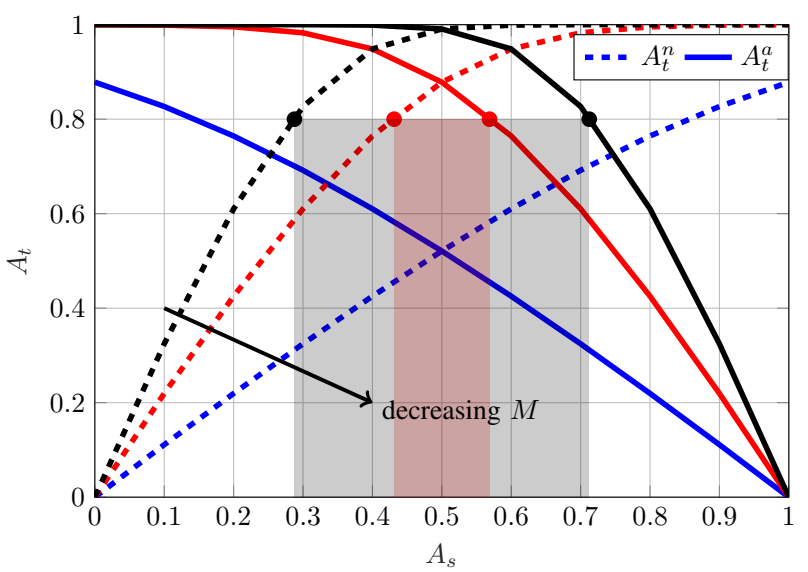

Fig. 7: Steady state time availability as a function of spatial availability for varying numbers of channels $(M=(10,20$, 30)).

time availability requirement of 0.8 is marked for $M=20$ and $M=30$ curves. As expected, the range of $A_{s}$ meeting the imposed requirement is larger in the latter case. This means that, a sufficient amount of resources can guarantee the time availability performance of multiple service classes.

\section{CONCLUSION}

In this paper we proposed a unified framework of computing temporal and spatial availability for a RAT-agnostic system. A novel, service-relevant definition of spatial availability was introduced taking into account the probability to achieve a targeted SIR threshold with a given confidence level. Temporal availability was investigated considering a novel, space availability-driven channel access scheme based on the concept of channel provisioning, bringing up the coupled relation between spatial and temporal availability and reliability. The study is supported by numerical evaluation results which underline the impact of different system parameter values on space/time availability and time reliability, as well as the coupled nature of these metrics. Further research directions can be envisioned, focusing on the particularities of different RAN characteristics, the service traffic model, as well as the connectivity criteria and the allocation of other resources.

\section{ACKNOWLEDGMENT}

The research leading to these results has been performed under the framework of the Horizon 2020 project ONE5G (ICT-760809) receiving funds from the European Union.

\section{REFERENCES}

[1] 3GPP, "Ts 22.261 service requirements for next generation new services and markets," 3rd Generation Partnership Project (3GPP), v16.3.0, 2018.

[2] Next Generation Mobile Networks Alliance, "Recommendations for NGMN KPIs and requirements for 5G," 2016.

[3] P. Popovski, "Ultra-reliable communication in 5G wireless systems," in 1st International Conference on $5 G$ for Ubiquitous Connectivity, Nov. 2014, pp. 146-151.

[4] M. Bennis, M. Debbah, and H. V. Poor, "Ultra-reliable and low-latency wireless communication: Tail, risk and scale," CoRR, vol. abs/1801.01270, 2018. [Online]. Available: http://arxiv.org/abs/1801.01270

[5] "5G for connected industries and automation," 5G Alliance for Connected Industries and Automation, Tech. Rep., 2018.

[6] "An assessment of LTE-V2X (PC5) and 802.11p direct communications technologies for improved road safety in the EU," 5G Automotive Association, Tech. Rep., 2017.

[7] H. V. K. Mendis and F. Y. Li, "Achieving ultra reliable communication in 5G networks: A dependability perspective availability analysis in the space domain," IEEE Communications Letters, vol. 21, no. 9, pp. 20572060, Sep. 2017.

[8] I. A. M. Balapuwaduge and F. Y. Li, "A joint time-space domain analysis for ultra-reliable communication in 5G networks," International Conference on Communications, 2018.

[9] T. Hößler, L. Scheuvens, N. Franchi, M. Simsek, and G. P. Fettweis, "Applying reliability theory for future wireless communication networks," in 2017 IEEE 28th Annual International Symposium on Personal, Indoor, and Mobile Radio Communications (PIMRC), Oct. 2017, pp. 1-7.

[10] A. Birolini, Reliability Engineering: Theory and Practice. Springer, 2010.

[11] D. Shrestha, Z. Pang, and D. Dzung, "Precise clock synchronization in high performance wireless communication for time sensitive networking," IEEE Access, vol. 6, pp. 8944-8953, 2018.

[12] J. G. Andrews, F. Baccelli, and R. K. Ganti, "A tractable approach to coverage and rate in cellular networks," IEEE Transactions on Communications, vol. 59, no. 11, pp. 3122-3134, Nov. 2011.

[13] M. Haenggi, "The meta distribution of the SIR in Poisson bipolar and cellular networks," IEEE Transactions on Wireless Communications, vol. 15, no. 4, pp. 2577-2589, Apr. 2016.

[14] H. Mendis, "Ultra reliable communication in 5G networks: A dependability-based availability analysis in the space domain," Master's thesis, University of Agder, 2017.

[15] R. K. Ganti and J. G. Andrews, "Correlation of link outages in lowmobility spatial wireless networks," in 2010 Conference Record of the Forty Fourth Asilomar Conference on Signals, Systems and Computers, Nov. 2010, pp. 312-316.

[16] I. A. M. Balapuwaduge, F. Y. Li, and V. Pla, "System times and channel availability for secondary transmissions in CRNs: A dependabilitytheory-based analysis," IEEE Transactions on Vehicular Technology, vol. 66, no. 3, pp. 2771-2788, Mar. 2017.

[17] R. J. Boucherie and E. A. van Doom, "Uniformization for $\lambda$-positive Markov chains," Communications in Statistics. Stochastic Models, vol. 14, no. 1-2, pp. 171-186, 1998. 